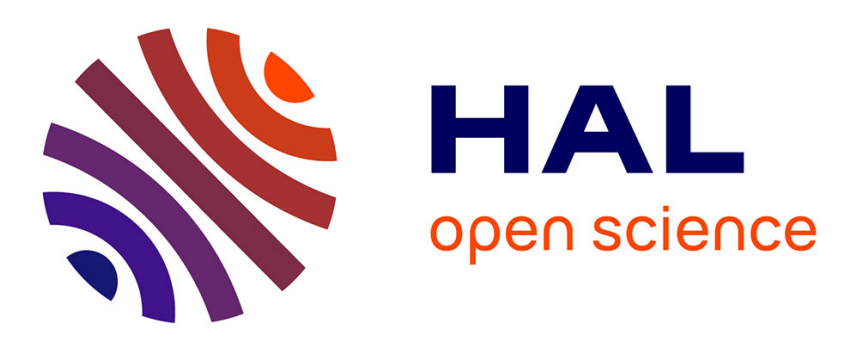

\title{
L'appropriation d'un outil de gestion de la qualité à travers le prisme de la légitimité: le cas d'un Institut d'Administration des Entreprises
}

\author{
Didier Bédé, Sébastien Bédé, Amélie Fiorello, Nathalie Maumon
}

\section{- To cite this version:}

Didier Bédé, Sébastien Bédé, Amélie Fiorello, Nathalie Maumon. L'appropriation d'un outil de gestion de la qualité à travers le prisme de la légitimité: le cas d'un Institut d'Administration des Entreprises. Revue management \& avenir, 2012, 4 (54), pp.83-106. 10.3917/mav.054.0083 . hal-00951382

\author{
HAL Id: hal-00951382 \\ https://hal.science/hal-00951382
}

Submitted on 24 Feb 2014

HAL is a multi-disciplinary open access archive for the deposit and dissemination of scientific research documents, whether they are published or not. The documents may come from teaching and research institutions in France or abroad, or from public or private research centers.
L'archive ouverte pluridisciplinaire HAL, est destinée au dépôt et à la diffusion de documents scientifiques de niveau recherche, publiés ou non, émanant des établissements d'enseignement et de recherche français ou étrangers, des laboratoires publics ou privés. 


\title{
L'appropriation d'un outil de gestion de la qualité à travers le prisme de la légitimité : le cas d'un Institut d'Administration des Entreprises
}

\author{
par Didier Bédé ${ }^{1}$, Sébastien Bédé2, Amélie Fiorello ${ }^{3}$ et Nathalie Maumon ${ }^{4}$
}

\section{Résumé}

L'amélioration continue $\mathrm{du}$ fonctionnement d'une organisation repose en partie sur l'appropriation des nouveaux outils de gestion par ses utilisateurs. L'appropriation est un processus dans lequel les acteurs vont interpréter, négocier et créer du sens. Autrement dit, les acteurs vont transformer les finalités de l'outil de gestion par l'utilisation qu'ils vont en faire et remettre en question les modèles de l'action collective (Grimand, 2006).

Cet article représente un premier niveau de questionnement sur l'appropriation d'un outil de gestion par les acteurs à travers la légitimité. Nous proposons d'analyser les formes de légitimité telles que définie par Suchman (1995) permettant de favoriser l'adoption d'un nouvel outil de gestion par différents groupes au sein d'une organisation publique. L'objet retenu pour cette recherche exploratoire est la démarche qualité des Instituts d'Administration des Entreprises (IAE) nommée Qualicert. Des entretiens de groupe ont été menés avec les parties prenantes internes d'un IAE. À partir des résultats nous proposerons une série d'applications managériales qui devront permettre à d'autres équipes dirigeantes de préparer la mise en place et la pérennisation de l'outil au sein de leur organisation.

Mots-clés : appropriation, outil de gestion, légitimité, management de la qualité

\section{Quality management tool adoption through the legitimacy prism: the case of French Institute of Business Administration}

\author{
par Didier Bédé ${ }^{1}$, Sébastien Bédé², Amélie Fiorello ${ }^{3}$ et Nathalie Maumon ${ }^{4}$
}

\begin{abstract}
Continuous improvement is partially built on new management tools user adoption. Adoption is a process in which individuals interpret, negotiate, and create meaning. In other words, management tools primary purposes are changed by the users and call into question models of collective action (Grimand, 2006). This study is a first step to question the adoption of quality management tool. We propose to analyze legitimacy forms as defined by Suchman (1995) to enhance new management tool adoption. These exploratory research deals with a quality certification named Qualicert for Institute of Business Administration in France. Focus group interviews had been conducted with each part of the organization. According to the results we put forward managerial levers to implement new management tool in a sustainable way.
\end{abstract}

Key words: adoption, management tool, legitimacy, quality management

\footnotetext{
1 Didier Bédé, ATER, Université Nice - Sophia Antipolis, Laboratoire GREDEG, UMR 7321 CNRS, bede@unice.fr

${ }^{2}$ Sébastien Bédé, Doctorant, Université Nice - Sophia Antipolis, Laboratoire GRM, EA 4711, sbede@unice.fr

${ }^{3}$ Amélie Fiorello, ATER, Université Nice - Sophia Antipolis, Laboratoire GRM, EA 4711, fiorello@ unice.fr

${ }^{4}$ Nathalie Maumon, Doctorante contractuelle chargée d'enseignement, Université Nice - Sophia Antipolis, Laboratoire GRM, EA 4711, nathalie.maumon@unice.fr
} 


\section{Introduction}

Les outils de gestion sont d'un usage courant dans les entreprises, toutefois, ils n'ont fait l'objet d'études universitaires que très récemment (Grimand, 2006). Aujourd'hui, la multiplication des outils dans le secteur privé mais aussi dans le secteur public, conduit les praticiens et les chercheurs à se pencher davantage sur la question de l'appropriation de ces outils. En effet, ceux-ci ne sont efficaces que si les individus concernés par leur application, c'est-à-dire ceux qui doivent les mettre en pratique, les utiliser dans leur travail quotidien, se les approprient. Or, dans certains cas particulier, tel que la certification qualité des Instituts d'Administration des Entreprises en France, l'approche retenue est essentiellement descendante, c'est-à-dire imposée par le réseau des IAE. La question de l'appropriation des outils de gestion est alors un enjeu majeur.

Cette recherche est un travail préalable à la construction sociale d'un outil de gestion et représente un premier niveau de questionnement sur la compréhension de la légitimité par les acteurs. L'objectif de cet article est double. L'objectif premier est de comprendre, dans un contexte organisationnel particulier tel qu'un institut universitaire, la légitimité d'un outil de management de la qualité par les différentes parties prenantes internes concernées par cet outil. Le second objectif est de proposer une série d'applications pratiques permettant de faciliter l'appropriation d'un outil de gestion.

Pour cela, nous allons dans un premier temps définir le concept d'outil de gestion et plus précisément du management de la qualité puis nous aborderons son appropriation à travers le cadre théorique de la légitimité. Dans un second temps, à travers un cas illustratif, nous tenterons de faire émerger les différents types de légitimité ayant favorisé l'appropriation d'un outil de gestion de la qualité par chaque service d'un Institut d'Administration des Entreprises à partir d'une série d'entretiens de groupes. Ces entretiens de groupe permettront de proposer une série d'applications pratiques afin d'aider les décideurs lors de la mise en place d'outil de gestion.

\section{L'appropriation d'un outil de management de la qualité : cadre théorique}

Nous allons dans cette première partie définir les concepts de base à savoir les outils de gestion et leur appropriation. Cette recherche s'inscrit dans la perspective des travaux de Grimand (2006), Hatchuel et Weil (1992) ou De Vaujany (2006) qui pensent qu'on ne peut appréhender l'appropriation d'un outil à travers une perspective technocentrée. Les relations hiérarchiques et la spécificité des statuts des acteurs concernés par l'outil et sa mise en pratique, nous conduisent à penser que l'appropriation est un processus cognitif qui peut passer par une construction de la légitimité de l'outil pour chaque partie prenante.

\subsection{Qualicert : un outil de gestion de la qualité dans les instituts d'enseignement et de recherche}

La conceptualisation de l'outil de gestion reste encore un sujet de débat dans le domaine des sciences de gestion où s'opposent une vision purement instrumentale et « technocentrée » de l'outil et une vision cognitive davantage basée sur des processus psychologiques de construction de sens et d'action collective. S'il n'existe pas de définition universelle de l'outil de gestion, nous pensons à l'instar de certains auteurs que l'outil de gestion est une entité mixte qui ne se limite pas à son support concret (Grimand, 2006 ; Hatchuel et Weil, 1992 ; De Vaujany, 2006). Selon Hatchuel et Weil (1992) l'outil de gestion est le fruit de trois éléments en interaction : un substrat technique, c'est-à-dire l'abstraction sur laquelle repose l'outil et qui lui permet de fonctionner ; une philosophie gestionnaire qui peut se traduire par les comportements de travail que l'outil est censé promouvoir, soit en les organisant, soit en incitant à leur adoption et enfin une vision simplifiée des relations organisationnelles qui 
définit « la scène » et les participants, l'outil établissant alors les règles du jeu dans un contexte déjà soumis à des règles plus ou moins strictes. Cette vision des relations permet de distinguer les acteurs influencés par l'outil parce qu'ils l'utilisent ou parce qu'ils se conforment à ses prescriptions; et les spécialistes de l'outil qui l'ont conçu et en assurent la diffusion. Ainsi, l'outil de gestion représente les artefacts matériels et symboliques mais aussi des registres d'action qui vont lui donner du sens (Grimand, 2006).

Dans la pratique, les outils de gestion sont nombreux et variés. Ils touchent toutes les fonctions des organisations et se traduisent dans la réalité par des tableaux de bord, des progiciels de gestion intégrés, des référentiels de compétences, des nouvelles règles comptables, des Intranets, des démarches qualité, etc. Aujourd'hui, la managérialisation de la sphère publique (Djélic, 2004) conduit à transférer des outils de gestion issus du privé vers des organisations publiques. C'est dans ce cadre que l'on assiste depuis quelque temps à la mise en place de démarches qualité au sein d'instituts universitaires de recherche et d'enseignement. C'est notamment le cas des Instituts d'Administration des Entreprises (IAE) en France qui ont désormais l'obligation d'être engagés dans un processus de certification de qualité de service : la certification «Qualicert ». Qualicert est donc un outil de gestion et plus précisément un outil d'évaluation des instituts universitaires spécialisés dans les sciences de gestion. Il est géré par l'entreprise SGS et mis en place dans les IAE à l'initiative de la direction, généralement aidée pour la mise en place de ce processus par un responsable qualité. Le référentiel utilisé pour la certification résulte de travaux réalisés par des représentants de l'Université au niveau national et européen. Il détaille les pièces à tenir à jour et les procédures à mettre en place afin d'atteindre les caractéristiques requises. Ce référentiel est garant de la qualité des instituts au regard de leurs engagements pris envers les étudiants, les partenaires de l'Université et les autorités de tutelles. In fine, la certification obtenue atteste de la qualité de services des instituts en termes de valeur ajoutée des formations, de qualité pédagogique et scientifique des équipes, d'efficacité de l'organisation administrative, de performance en matière de partenariats avec des entreprises et d'ouverture internationale.

La certification qualité est "l'attestation de conformité d'une entité à des normes, par un organisme tiers »(Grenard, 1996, p.45). Cette attestation s'obtient lorsque l'organisation a mis en place des outils de management de la qualité c'est-à-dire des dispositifs qui permettent «à une entreprise, une organisation de gérer ses processus ou ses activités, de sorte que ses produits ou services répondent aux objectifs qu'elle s'est fixés » en matière de qualité (Rolland, 2009, p. 33). Dans cette situation, le management est normalisé et la norme fournit un modèle à suivre dans la mise en place et le fonctionnement de ce système. Elle est aussi à la base de la production de documents de référence, le substrat technique sur lequel repose l'outil de management de la qualité. Le référentiel est un document important, il se définit comme « un cadre partagé pour l'exercice d'une activité »(Chevalier, 2009, p.220). La certification fait systématiquement intervenir un organisme tiers et indépendant, ce qui permet d'obtenir une plus grande confiance dans l'obtention de cette certification (Grenard, 1996).

Les outils de management de la qualité, de la même façon que l'ensemble des outils de gestion, dans le secteur privé comme dans le secteur public, permettent de formaliser l'action organisée (David, 1998) et ainsi de rationaliser les processus dans une organisation. Ils apportent une logique, une rigueur et une simplification censée permettre d'atteindre une meilleure efficacité et une performance optimale. Toutefois, le succès d'un outil de gestion, quel qu'il soit, dépend fortement de son appropriation par les acteurs qui doivent le mettre en pratique. Ainsi, le contexte joue un rôle prépondérant. En effet, l'outil subit généralement des transformations successives au fur et à mesure de sa diffusion dans l'organisation par le biais d'un processus d'appropriation des acteurs. 


\subsection{Analyser l'appropriation des outils de gestion à travers la légitimité}

L'appropriation des outils de gestion est devenue un enjeu majeur compte tenu de la multiplication de ces derniers (De Vaujany, 2006). Pour Grimand, l'appropriation est «un processus interprétatif, de négociation et de construction du sens à l'intérieur duquel les acteurs questionnent, élaborent, réinventent les modèles de l'action collective » (2006, p.17).

Se poser la question de l'appropriation des outils de gestion revient à se demander ce que deviennent ces outils dans les mains de ceux qui les utilisent et comment ils sont rendus propres à l'usage. Plus précisément, il s'agit de se demander comment les individus s'approprient ces outils et surtout comment faciliter ce processus d'appropriation, essentiel au succès de l'outil (De Vaujany, 2006).

En suivant les travaux de Buisson (2006), nous pensons qu'une appropriation réussie d'un outil de gestion passe par sa légitimation en interne. Cette légitimation représente alors «un processus complexe de nature à la fois symbolique, cognitive, identitaire » (Buisson, 2006, p.157). En mobilisant la théorie néo-institutionnelle, Buisson nous apprend que pour légitimer un outil de gestion il faut désigner un «entrepreneur institutionnel», une sorte de « légitimateur», qui aura pour rôle de promouvoir et légitimer les nouvelles pratiques mais aussi de mobiliser les acteurs internes et externes concernés par les pratiques en question. Plus exactement, "il doit promouvoir son projet et ses idées auprès des différentes parties prenantes, et les rendre acceptables et proches des préoccupations de ces audiences, bien qu'elles soient multiples » (op.cit., p.164). Pour cela, il peut mobiliser différentes ressources et peut agir sur des aspects tangibles des organisations mais aussi sur des aspects intangibles tels que les symboles, les croyances, les idéologies, les rites et les mythes (Pettigrew, 1979). La légitimité étant socialement construite, les aspects symboliques et cognitifs sont donc essentiels, et généralement, la promotion des nouvelles pratiques se fait par le biais de stratégies informationnelles et discursives. Ces stratégies doivent déboucher sur la légitimation des pratiques et sur leur intégration dans les structures et les croyances organisationnelles. Il convient donc de repérer et de distinguer les différentes parties prenantes internes concernées par l'outil et de favoriser son appropriation en jouant sur des symboles évocateurs partagés (Laufer, 2008) dans le but d'obtenir l'appropriation et l'acceptation de l'outil.

Ainsi, pour appréhender la construction sociale de la légitimité qui s'établit autour d'un outil de gestion, la voie privilégiée est l'analyse du discours des acteurs (Phillips, Lawrence et Hardy, 2004). C'est ce que nous proposons de faire dans cet article. En effet, nous souhaitons savoir comment l'appropriation d'un outil de gestion de la qualité s'est opérée au sein d'un institut universitaire à travers la construction sociale de sa légitimité pour différentes parties prenantes. Dans ce cas particulier, les légitimateurs à l'origine de la nouvelle pratique sont les directeurs des IAE épaulés par les responsables qualité en interne. Pour faciliter l'appropriation de l'outil, ces derniers doivent trouver comment faciliter la construction de la légitimité d'un tel outil pour chaque partie prenante concernée, en l'occurrence : l'équipe de direction de la structure, les directeurs de formation, les services généraux, les responsables des relations internationales et les secrétaires pédagogiques.

Dans cet article, nous proposons d'étudier un IAE qui a déjà mis en place avec succès l'outil Qualicert et d'analyser les discours des différentes parties prenantes afin de comprendre comment s'est construite la légitimité de l'outil et comment celle-ci a favorisé son appropriation. Pour cela, nous choisissons de mobiliser le cadre théorique proposé par Suchman (1995) qui représente une synthèse entre l'approche néo-institutionnelle et l'approche stratégique de la légitimité. Ce cadre permet de concilier le point de vue des acteurs de l'organisation, dont le manager est la figure centrale, avec des éléments de la culture d'entreprise en tant que facteurs essentiels de la vie des organisations. 
L'approche stratégique (Oliver, 1991) perçoit la légitimité à travers une perspective managériale, la légitimité y est instrumentale. Elle est appréhendée comme un processus actif, permanent ou temporaire, poursuivi ou délibérément ignoré par l'organisation. Les approches institutionnelle et néo-institutionnelle (Parsons, 1960 ; DiMaggio et Powell, 1983 ; 1991) perçoivent, quant à elles, la légitimité comme une fin poursuivie naturellement par toutes les organisations et un processus continu s'approchant d'un processus d'institutionnalisation.

Combinant les deux perspectives, Suchman (1995) définit la légitimité comme une "perception généralisée ou supposition selon laquelle les actions d'une entité sont désirables, et appropriées au sein d'un système socialement construit de normes, de valeurs, de croyances et de règles partagées ». À partir de cette définition, il souligne que la légitimité peut être de trois ordres : pragmatique, moral et cognitif. Nous nous intéresserons ici aux différents types de légitimité qui peuvent se construire autour de l'outil et non de l'organisation en général.

La légitimité pragmatique repose sur l'intérêt procuré aux cibles les plus immédiates de l'organisation et donc sur une évaluation des bénéfices individuels tirés de l'outil, ainsi celuici est jugé légitime s'il procure des bénéfices à l'audience ciblée. La légitimité pragmatique peut être obtenue à travers les échanges avec les parties prenantes, elle reposera alors sur la satisfaction des besoins et les parties prenantes décideront si les échanges sont avantageux ou non. Elle peut aussi être obtenue à travers l'influence exercée sur celles-ci. Dans ce second cas, il s'agira de savoir si les parties prenantes ont été intégrées dans la phase de mise en place de l'outil. Enfin, la légitimité pragmatique peut être d'ordre dispositionnel. La légitimité dispositionnelle repose sur le fait que les individus ont tendance à personnifier les choses et à leur accorder soit de la bienveillance, soit de la malveillance, ainsi qu'une bonne ou une mauvaise réputation.

La légitimité peut également être d'ordre moral. Elle se rapproche alors de la légitimité telle que définie par l'approche institutionnelle et se rapporte à une «évaluation normative positive de l'organisation et de ses activités » (Suchman, 1995, p.579). Elle relève moins du jugement relatif aux bénéfices de l'outil pour l'évaluateur que de savoir s'il s'agit de « la bonne chose à faire ». Les parties prenantes n'attendent pas des bénéfices immédiats mais attendent que l'outil contribue au bien-être sociétal ou au bien-être de l'organisation dans son ensemble tel que défini par un système de valeur socialement construit. La légitimité morale s'étudie à travers l'évaluation des résultats et des conséquences, des techniques et des procédures et des structures mais aussi des personnes. La légitimité conséquentielle cherche à établir, de façon rationnelle, la légitimité des résultats et des conséquences de l'outil. La légitimité procédurale cherche à établir si les moyens ou les différentes procédures qui découlent de la mise en place de l'outil sont socialement adaptés. Ce type de légitimité est d'autant plus important en l'absence de résultats clairement et facilement mesurables (Scott, 1992). La légitimité morale peut également s'étudier au travers de la légitimité structurelle qui repose sur l'évaluation de l'adoption de structures institutionnellement prescrites. Elle revient à se demander si l'outil permet d'atteindre des buts collectifs de façon appropriée et adéquate. En dernier lieu, la légitimité morale peut porter sur la légitimité personnelle. Elle repose alors sur le charisme des leaders et des individus à l'origine de la mise en place de nouvelles pratiques. La légitimité personnelle est rare, transitoire et idiosyncratique.

Finalement, la légitimité cognitive, moins explicite que les deux types de légitimités présentées ci-dessus, relève de l'acceptation d'un fait ou d'une information présentée comme allant de soi (De Benedetti et Philippe, 2011) ou considérée comme acquis, ce que Suchman (1995) nomme «taken-for-grantedness » et que nous traduisons par «légitimité explicite ». Cette acceptation sera d'autant plus grande que le fait ou l'information est institutionnalisé et connu. La légitimité cognitive repose sur la compréhensibilité et l'explicitation. La légitimité basée sur la compréhensibilité repose sur la disponibilité des modèles culturels qui permettent 
de donner des explications plausibles aux procédures et à l'existence de l'outil. Enfin, la légitimité explicite repose sur l'institutionnalisation de l'outil qui lui permettra de perdurer au-delà de ses créateurs.

Le cadre théorique que nous venons d'exposer va nous permettre d'analyser l'appropriation d'un outil de management de la qualité dans un Institut d'Administration des Entreprises. Pour cela, nous adoptons une démarche qualitative fondée sur une série d'entretiens collectifs.

\section{Méthodologie}

L'article proposé s'appuie sur un cas illustratif au sens de David (2003). Il sert à illustrer le cadre théorique mobilisé et à vérifier qu'il est opérationnalisable sans usage démonstratif ni, $a$ priori, général. D'autre part, la position adoptée par les chercheurs est multiple. L'un des coauteurs, en tant que responsable de la certification qualité de l'IAE, a une position de participant-observateur. Cette position de praticien qui engendre « une réactivité de la part des sujets [interrogés] car il est mandaté au sein de l'organisation » (Thiétart, 2007, p.245) est relativisée par la position des autres chercheurs qui, en tant qu'observateur-participants, disposent d'une plus grande liberté pour compléter les observations et ont une plus grande neutralité à l'égard des sujets interrogés.

Par ailleurs, l'entretien collectif est une méthode qui s'est généralisée car elle peut être adaptée de multiples façons en fonction des objectifs à atteindre. L'efficacité de cette méthode discursive réside dans son rôle de catalyseur de l'expérience vécue par l'ensemble des enquêtés (Duchesne et Haegel, 2008). La recherche menée dans cet article considère au contraire la discussion entre les participants, en tant que forme primaire de données collectées, comme l'intérêt principal de la recherche (Morgan, 1997). En l'occurrence, il s'agit des discours sur la légitimité d'un outil de gestion reconnu par différentes parties prenantes au sein d'un institut public d'enseignement supérieur.

\subsection{Collecte des données}

La visée exploratoire de notre article implique que les participants soient relativement libres de discuter du thème. Néanmoins, pour des raisons de modération et de contrôle de la discussion par l'interviewer, un guide d'entretien semi-directif a été construit. Celui comprend 16 questions qui portent sur quatre thématiques: la vision de l'Institut; la légitimité pragmatique ; la légitimité morale et la légitimité cognitive. Chaque thématique dans le guide d'entretien contient des questions de relance permettant d'engager la discussion sur un soustype de légitimité issu du cadre théorique de notre étude.

L'interviewer n'a pas eu pour objectif d'obtenir un consensus autour du sujet mais a eu pour rôle de dynamiser la discussion, d'éviter qu'un individu ou une petite coalition domine le groupe, d'encourager les sujets récalcitrants à participer et d'obtenir du groupe une analyse la plus complète possible du thème abordé (Fontana et Frey, 1994; Merton, Lowenthal et Kendall, 1990).

Notre échantillon est divisé en cinq sous-groupes afin de répondre à l'hétérogénéité des parties prenantes internes à l'organisation en relation avec la certification Qualicert (Tableau 1, p.7). La détermination de l'échantillon des répondants ne s'est pas faite au hasard: «dans les recherches qualitatives, la sélection des répondants n'est pas aléatoire, le chercheur vise plutôt à avoir accès, à l'intérieur d'un échantillon modeste de répondants, à la diversité des points de vue sur le sujet à l'étude »(Demers, 2003, p.191).

Thiétart (2007, p.243) souligne néanmoins que «l'authenticité des discours est sujette à caution, tant les jeux de pouvoir et les enjeux du devenir des sujets au sein de l'organisation peuvent intervenir ». 
Tableau 1 - La catégorisation de l'échantillon

\begin{tabular}{|c|c|c|c|}
\hline \multirow[t]{4}{*}{ Groupe 1} & \multirow{4}{*}{$\begin{array}{l}\text { Direction de la structure } \\
\text { (DirStr) }\end{array}$} & Directeur & \multirow{4}{*}{4 individus } \\
\hline & & Directeur adjoint aux relations entreprises & \\
\hline & & Directeur adjoint à la pédagogie & \\
\hline & & Directrice administrative & \\
\hline \multirow[t]{2}{*}{ Groupe 2} & \multirow{2}{*}{$\begin{array}{l}\text { Directeurs de formation } \\
\text { (DirFor) }\end{array}$} & Directrice M2 Communication & \multirow{2}{*}{2 individus } \\
\hline & & Directeur L3 Management & \\
\hline \multirow[t]{3}{*}{ Groupe 3} & \multirow{3}{*}{$\begin{array}{l}\text { Services généraux } \\
\text { (SerGen) }\end{array}$} & Assistante de Direction & \multirow{3}{*}{3 individus } \\
\hline & & Chargée de communication & \\
\hline & & Chargée du système d'information & \\
\hline \multirow[t]{3}{*}{ Groupe 4} & \multirow{3}{*}{$\begin{array}{l}\text { Pôle des relations } \\
\text { internationales } \\
\text { (RI) }\end{array}$} & Responsable & \multirow{3}{*}{3 individus } \\
\hline & & Secrétaire & \\
\hline & & Secrétaire & \\
\hline \multirow[t]{4}{*}{ Groupe 5} & \multirow{3}{*}{$\begin{array}{l}\text { Secrétaires pédagogiques } \\
\text { (SP) }\end{array}$} & Secrétaire & \multirow{3}{*}{3 individus } \\
\hline & & Secrétaire & \\
\hline & & Secrétaire & \\
\hline & & Total & 15 individus \\
\hline
\end{tabular}

En ce sens, l'entretien collectif est une méthode qui «prend en compte le contexte mais tend à réduire l'impact de la hiérarchisation que suppose la division du travail d'interrogation » (Duchesne et Haegel, 2008, p.33). De plus, l'homogénéisation par sous-groupes de notre échantillon, en regroupant ensemble des individus qui partagent des valeurs similaires, a eu pour motivation première de créer un effet libérateur (Johnson, 1996) et une opportunité pour les participants de s'engager dans une conversation significative sur la légitimité d'un outil de gestion en relativisant les biais engendrés.

\subsection{Analyse des données}

L'analyse des données recueillies a nécessité d'opérer une classification thématique et analytique des corpus, de réaliser une codification puis d'entreprendre des croisements pour comparer certaines unités du discours. Ces différentes étapes participent à ce que Deschenaux (2007) nomme le «principe de déstructuration-restructuration » d'un corpus qui consiste à décontextualiser le corpus global puis à le regrouper en unité de sens.

Les corpus recueillis lors des entretiens ont donc été analysés groupe par groupe. Les différentes questions sont liées à chaque type de légitimité spécifique et suivent le cadre théorique. L'étape suivante a consisté à traduire les questions en codes en suivant la structure originale du corpus. Un codage en double aveugle des corpus, facilité par l'utilisation du logiciel Nvivo, a ensuite permis d'affiner le classement des données en fonction d'une interprétation de chaque chercheur et d'une comparaison des nouveaux codages effectués par une décontextualisation du corpus. Les textes codés ont ensuite été restructurés au sein d'un fichier unique en fonction des groupes et des types de légitimités abordés.

Le corpus recueilli suite à la collecte de données a fait l'objet d'une première analyse consistant à vérifier la présence des différents types de légitimité pour chacun des entretiens de groupe réalisés. La présence de chaque type de légitimité (pragmatique - morale cognitive) pour chaque groupe interrogé renforce la pertinence du cadre théorique utilisé dans son approche globale. Toutefois, dans une approche plus fine, on constate que le groupe du pôle des relations internationales ne mentionne pas les légitimités d'échange, d'influence, conséquentielle, procédurale et structurelle. Les directeurs de formation ne mentionnent pas les légitimités dispositionnelle, et explicite. Les autres groupes contribuent tous à la compréhension des différents types et sous-types de légitimité, seule la légitimité dispositionnelle n'est mentionnée que par un seul groupe.

Pour affiner notre compréhension de l'appropriation, à travers le prisme de la légitimité, d'un outil de gestion de la qualité par les parties prenantes internes d'un Institut d'Administration 
des Entreprises, nous avons réalisé une analyse qualitative des corpus dont nous présentons ci-après les principaux résultats.

\section{La légitimité de Qualicert au sein d'un Institut d'Administration des Entreprises}

Nous allons voir dans cette partie comment les parties prenantes internes d'un IAE ont construit la légitimité d'un outil de management de la qualité. Nous présentons ici les analyses des discours par grands types de légitimité. Plus précisément, pour chaque partie prenante, nous exposons les verbatims les plus pertinents liés aux différents sous-types de la légitimité pragmatique.

\subsection{La légitimité pragmatique}

Nous traiterons dans cette partie de la légitimité pragmatique qui regroupe les trois sous-types de légitimités suivants : la légitimité d'échange, d'influence et dispositionnelle.

Ci-dessous, nous présentons le tableau relatif à la légitimité d'échange (Tableau 2, p.8).

Tableau 2 - Synthèse de la légitimité d'échange

\begin{tabular}{|c|c|c|c|c|c|}
\hline & $\begin{array}{c}\text { Groupe } 1 \\
\text { DirStr }\end{array}$ & $\begin{array}{c}\text { Groupe } 2 \\
\text { DirFor }\end{array}$ & $\begin{array}{c}\text { Groupe } 3 \\
\text { RI }\end{array}$ & $\begin{array}{c}\text { Groupe } 4 \\
\text { SerGen }\end{array}$ & $\begin{array}{c}\text { Groupe } 5 \\
\text { SP }\end{array}$ \\
\hline 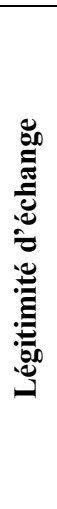 & $\begin{array}{c}\text { «On parle d'équipe depuis } \\
\text { Qualicert ». } \\
\text { "Une cohésion s'est } \\
\text { créée ». } \\
\text { «J'applique (le référentiel) } \\
\text { et le message que je passe } \\
\text { aux équipes: "ben voilà on } \\
\text { a un référentiel, on doit } \\
\text { appliquer le référentiel, on } \\
\text { n'a pas le choix". Alors } \\
\text { qu'avant c'était: "oh les } \\
\text { caprices de Mme T." ». }\end{array}$ & $\begin{array}{c}\text { «J'avais deux } \\
\text { cours où cela s'est } \\
\text { très mal passé } \\
\text { l'année dernière. } \\
\text { J'ai changé } \\
\text { d'intervenant [...] } \\
\text { c'est grâce à } \\
\text { Qualicert que j'ai } \\
\text { su ». } \\
\text { « Je m'en suis } \\
\text { servie auprès d'un } \\
\text { intervenant [...]. } \\
\text { Après, c'est } \\
\text { délicat ». }\end{array}$ & & $\begin{array}{l}\text { «On peut se } \\
\text { référer au } \\
\text { référentiel, }[. . .] \\
\text { ça nous permet } \\
\text { d'avoir un } \\
\text { support pour } \\
\text { dire : "c'est } \\
\text { écrit, venez pas } \\
\text { nous chercher } \\
\text { sur ça" ». }\end{array}$ & $\begin{array}{l}\text { «J'ai resserré les vis } \\
\text { pour les conventions. On } \\
\text { a été plus vigilant. Je dis } \\
\text { aux directeurs "Attention } \\
\text { aux conventions de stage } \\
\text { car il faut que nous } \\
\text { puissions les retrouver } \\
\text { dans quelques années" ». } \\
\text { "Cela nous permet de } \\
\text { nous appuyer sur quelque } \\
\text { chose, notamment devant } \\
\text { les enseignants ». }\end{array}$ \\
\hline
\end{tabular}

Nous pouvons voir que la légitimité d'échange de l'outil Qualicert est mentionnée par toutes les parties prenantes excepté le groupe des relations internationales.

Le groupe de la direction de la structure mentionne que les consignes qu'il fait passer sont acceptées plus facilement lorsqu'elles sont issues du référentiel Qualicert. Pour ce groupe, l'outil de gestion a soudé l'ensemble de l'IAE, notamment grâce à la communication faite autour de Qualicert. Pour les directeurs de formation, la légitimité d'échange passe essentiellement par le rapport qu'ils entretiennent avec les étudiants et les intervenants. Les conseils de perfectionnement, qui synthétise le point de vue des étudiants sur les améliorations à apporter à la formation, favorisent la légitimité des décisions prises par les directeurs de formations et améliorent leurs relations avec les enseignants. Pour les services généraux et les secrétaires pédagogiques, Qualicert permet de justifier et d'appuyer les demandes faites aux divers interlocuteurs rencontrés dans leur travail quotidien, à savoir les étudiants, les intervenants et une partie des enseignants titulaires.

Enfin, pour le groupe des relations internationales, la couverture nationale de Qualicert ne leur permet pas d'obtenir une légitimité d'échange auprès des étudiants internationaux.

Ainsi, de façon générale et à l'exception du groupe des relations internationales, l'ensemble des services de l'IAE reconnaît le rôle de facilitateur d'échanges exercé par Qualicert. 
La légitimité morale repose également sur la légitimité d'influence. Celle-ci permet d'analyser quels sont les leviers qui permettent d'intégrer les parties prenantes dans la stratégie de l'entreprise et dans la mise en place de la démarche qualité (Tableau 3, p.9).

Tableau 3 - Synthèse de la légitimité d'influence

\begin{tabular}{|c|c|c|c|c|c|}
\hline & $\begin{array}{c}\text { Groupe } 1 \\
\text { DirStr }\end{array}$ & $\begin{array}{c}\text { Groupe } 2 \\
\text { DirFor }\end{array}$ & $\begin{array}{c}\text { Groupe } 3 \\
\text { RI }\end{array}$ & $\begin{array}{c}\text { Groupe } 4 \\
\text { SerGen }\end{array}$ & $\begin{array}{c}\text { Groupe } 5 \\
\text { SP }\end{array}$ \\
\hline 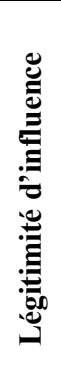 & $\begin{array}{l}\text { «C'est-à-dire que } \\
\text { quand on avait une } \\
\text { procédure à mettre en } \\
\text { place, je ne décidais } \\
\text { pas toute seule dans } \\
\text { mon coin. Je disais : } \\
\text { "ben voilà, vous, vous } \\
\text { êtes sur le terrain, } \\
\text { comment vous pensez } \\
\text { que ce serait au } \\
\text { mieux?" ». }\end{array}$ & $\begin{array}{l}\text { «Cela a été présenté, } \\
\text { expliqué et imposé. } \\
\text { Dans la mise en œuvre, } \\
\text { cela s'est fait au } \\
\text { travers des réunions. } \\
\text { Cela s'est fait au } \\
\text { travers de mailings ». } \\
\text { «C'est plus un } \\
\text { engagement personnel, } \\
\text { pour les étudiants } \\
\text { avant tout». }\end{array}$ & & $\begin{array}{c}\text { «Ce n'est pas qu'il ne } \\
\text { prenne pas ma voix en } \\
\text { considération, ils vont } \\
\text { la prendre quand c'est } \\
\text { lié à la communication. } \\
\text { On distingue bien } \\
\text { l'administration et la } \\
\text { pédagogie, je ne fais } \\
\text { pas partie de la } \\
\text { pédagogie ». }\end{array}$ & $\begin{array}{c}\text { «[...] pour une fois } \\
\text { on se sent impliqué. } \\
\text { Parce qu'on a } \\
\text { toujours } \\
\text { l'impression d'être } \\
\text { des pions et on ne } \\
\text { nous demande pas } \\
\text { notre avis. Et là, on } \\
\text { ne s'est pas senties } \\
\text { manipulées ». }\end{array}$ \\
\hline
\end{tabular}

Nous voyons à travers ce tableau que la direction de la structure, qui est à l'origine de la démarche, a fait preuve d'une volonté d'intégrer les différentes parties prenantes internes lors de la mise en place de l'outil mais aussi lors des phases d'amélioration. Cette volonté a ainsi favorisé la légitimité d'influence pour la quasi-totalité des parties prenantes. En effet, les responsables des relations internationales n'ont pas eu le sentiment d'avoir été intégré en amont à la mise en place de l'outil : "Je n'ai pas entendu parler de Qualicert jusqu'à la première réunion avec le responsable pour préparer l'audit. Enfin, j'en ai peut-être entendu parler, mais je ne savais pas ce que c'était ».

La démarche qualité semble avoir été suivie par les directeurs de formation moins par obligation que par conscience professionnelle. À travers leurs discours, on comprend qu'ils ont été informés mais pas totalement intégrés dans la phase de mise en place de l'outil.

Enfin, les responsables des services généraux et les secrétaires pédagogiques reconnaissent leur influence lors de la mise en place de l'outil et nous indiquent que leur pouvoir de décision était lié à leur domaine de compétences.

Pour en terminer avec la légitimité pragmatique, nous allons traiter de la légitimité dispositionnelle (Tableau 4, p.9). Ici, nous cherchons à savoir si les personnes interrogées ont tendance à personnifier l'outil de gestion Qualicert et à lui accorder des qualités « humaines ».

Tableau 4 - Synthèse de la légitimité dispositonnelle

\begin{tabular}{|c|c|c|c|c|}
\hline $\begin{array}{c}\text { Groupe 1 } \\
\text { DirStr }\end{array}$ & $\begin{array}{c}\text { Groupe 2 } \\
\text { DirFor }\end{array}$ & $\begin{array}{c}\text { Groupe 3 } \\
\text { RI }\end{array}$ & $\begin{array}{c}\text { Groupe 4 } \\
\text { SerGen }\end{array}$ & $\begin{array}{c}\text { Groupe 5 } \\
\text { SP }\end{array}$ \\
\hline
\end{tabular}

Nous voyons que la légitimité dispositionnelle n'a été mentionnée que par un seul groupe : celui des relations internationales. Ce dernier accorde à Qualicert un rôle de manager. Il semble ainsi que la légitimité dispositionnelle soit plus facile à percevoir lorsque l'on traite d'une organisation plutôt que d'un outil. 


\subsection{La légitimité morale}

Nous traiterons dans cette partie de la légitimité morale qui regroupe les quatre sous-types de légitimités suivants : la légitimité conséquentielle, procédurale, structurelle et personnelle.

Le Tableau 5, ci-dessous, présente les verbatims associés à la légitimité conséquentielle, c'està-dire liée aux résultats de la démarche Qualicert.

Tableau 5 - Synthèse de la légitimité conséquentielle

\begin{tabular}{|c|c|c|c|c|c|}
\hline & $\begin{array}{c}\text { Groupe } 1 \\
\text { DirStr }\end{array}$ & $\begin{array}{c}\text { Groupe } 2 \\
\text { DirFor }\end{array}$ & $\begin{array}{c}\text { Groupe } 3 \\
\text { RI }\end{array}$ & $\begin{array}{c}\text { Groupe } 4 \\
\text { SerGen }\end{array}$ & $\begin{array}{c}\text { Groupe } 5 \\
\text { SP }\end{array}$ \\
\hline & $\begin{array}{l}\text { «Qualicert ça nous amène à } \\
\text { respecter un cahier des } \\
\text { charges qui a été réfléchi en } \\
\text { amont avec le réseau des } \\
\text { IAE qui a souhaité qu'il y } \\
\text { ait un minimum de } \\
\text { procédures à tous les } \\
\text { niveaux ». } \\
\text { «Comme le maître mot } \\
\text { c'est la qualité, et bien ça } \\
\text { nous a amenés à mettre en } \\
\text { place des règles strictes de } \\
\text { non-compensation entre les } \\
\text { UE ». } \\
\text { «On arrive même à mettre } \\
\text { en place des choses qui vont } \\
\text { au-delà du référentiel ». }\end{array}$ & $\begin{array}{c}\text { « Cela sert à } \\
\text { préparer mon } \\
\text { conseil de } \\
\text { perfectionnement ». } \\
\text { «Qualicert c'est } \\
\text { avant tout, un } \\
\text { moyen de } \\
\text { formaliser ce qui est } \\
\text { fait, ce qui est à } \\
\text { faire, ce qui est à } \\
\text { améliorer ». }\end{array}$ & & $\begin{array}{c}\text { «Ça permet de } \\
\text { vendre l'IAE avec } \\
\text { un bonus pour les } \\
\text { étudiants. Il s'agit } \\
\text { d'une institution } \\
\text { d'éducation qui a } \\
\text { une certification de } \\
\text { qualité au niveau } \\
\text { des services ». } \\
\text { «Qualicert a permis } \\
\text { une meilleure } \\
\text { organisation. Dans } \\
\text { le fonctionnement } \\
\text { administratif ». }\end{array}$ & $\begin{array}{l}\text { «J'ai l'impression } \\
\text { que cela fixe un } \\
\text { cadre et que c'est } \\
\text { bien surtout dans } \\
\text { l'administration et } \\
\text { uniformise toutes } \\
\text { les procédures ». } \\
\text { « Ça a facilité notre } \\
\text { travail puisque tout } \\
\text { est posé ». } \\
\text { «C'est un gage de } \\
\text { qualité pour les } \\
\text { étudiants ». }\end{array}$ \\
\hline
\end{tabular}

La légitimité conséquentielle se réfère aux résultats concrets découlant de l'adoption de la démarche Qualicert. Ce tableau nous permet de voir que les directeurs de formations n'accordent pas une grande légitimité aux résultats de la démarche qualité tandis que les responsables des relations internationales ne la mentionnent pas du tout.

Pour la direction de la structure, Qualicert permet de respecter un cahier des charges rigoureux, commun à l'ensemble des IAE ayant adopté cette démarche. De plus, l'adoption de la démarche Qualicert semble être une passerelle vers un objectif de qualité totale, dépassant le référentiel initial. Le groupe de direction doit faire prendre conscience aux parties prenantes internes de l'intérêt de Qualicert et leur expliciter les résultats attendus de cette démarche.

De leur côté, les services généraux et les secrétaires pédagogiques apprécient les résultats de Qualicert tant au niveau interne, c'est-à-dire en termes d'optimisation de leur travail, qu'externe, c'est-à-dire en termes visibilité auprès des étudiants.

Outre les résultats, la légitimité morale porte aussi sur les moyens. Dans le cas de l'IAE et de l'adoption de Qualicert, nous faisons référence aux diverses procédures édictées par Qualicert (Tableau 6, p.10).

Tableau 6 - Synthèse de la légitimité procédurale

\begin{tabular}{|c|c|c|c|c|c|}
\hline & $\begin{array}{c}\text { Groupe } 1 \\
\text { DirStr }\end{array}$ & $\begin{array}{c}\text { Groupe } 2 \\
\text { DirFor }\end{array}$ & $\begin{array}{c}\text { Groupe } 3 \\
\text { RI }\end{array}$ & $\begin{array}{c}\text { Groupe } 4 \\
\text { SerGen }\end{array}$ & $\begin{array}{c}\text { Groupe } 5 \\
\text { SP }\end{array}$ \\
\hline 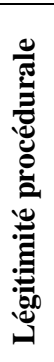 & $\begin{array}{c}\text { «Quand on met } \\
\text { quelque chose en place } \\
\text { on ne cherche pas } \\
\text { uniquement la } \\
\text { procédure, mais la } \\
\text { qualité de la } \\
\text { procédure ». } \\
\text { « On a le réflexe qualité } \\
\text { maintenant dans notre } \\
\text { travail ». }\end{array}$ & $\begin{array}{l}\text { « Après, même la } \\
\text { démarche est à } \\
\text { améliorer. De la } \\
\text { même façon qu'elle } \\
\text { améliore le travail, } \\
\text { l'organisation, il } \\
\text { faudra certainement } \\
\text { l'améliorer ». }\end{array}$ & & $\begin{array}{l}\text { «C'est mieux d'obliger } \\
\text { les gens à classer, que } \\
\text { tout le monde puisse } \\
\text { retrouver, que la } \\
\text { collègue puisse } \\
\text { retrouver les } \\
\text { documents ». } \\
\text { «Ça a permis qu'on } \\
\text { travaille tous dans le } \\
\text { même sens ». }\end{array}$ & $\begin{array}{c}\text { «Je fais attention à } \\
\text { ce que les } \\
\text { documents puissent } \\
\text { être lisibles. On se } \\
\text { le dit entre nous. } \\
\text { J'ai l'habitude } \\
\text { maintenant, s'il y a } \\
\text { un litige sur un } \\
\text { dossier je suis } \\
\text { organisé ». }\end{array}$ \\
\hline
\end{tabular}


Nous observons que la mise en place de nouvelles procédures, orientées vers une démarche qualité, est considérée comme légitime par quasiment toutes les parties prenantes sauf les responsables des relations internationales. Alors que la direction y voit la diffusion d'une démarche globale de la qualité, les responsables administratifs et les secrétaires pédagogiques insistent sur l'amélioration et l'efficacité des pratiques de travail.

Enfin, les directeurs de formation ne sont pas totalement convaincus de la légitimité des procédures Qualicert et pensent qu'elles doivent être constamment améliorées.

La légitimité morale porte également sur la légitimité structurelle (Tableau 7, p.11). Dans notre cas, il s'agit d'évaluer si l'IAE, dans le but de répondre aux attentes d'une démarche qualité, a adopté une structure adéquate du point de vue des parties prenantes internes.

Tableau 7 - Synthèse de la légitimité structurelle

\begin{tabular}{|c|c|c|c|c|c|}
\hline & $\begin{array}{c}\text { Groupe } 1 \\
\text { DirStr }\end{array}$ & $\begin{array}{c}\text { Groupe } 2 \\
\text { DirFor }\end{array}$ & $\begin{array}{c}\text { Groupe } 3 \\
\text { RI }\end{array}$ & $\begin{array}{c}\text { Groupe } 4 \\
\text { SerGen }\end{array}$ & $\begin{array}{c}\text { Groupe } 5 \\
\text { SP }\end{array}$ \\
\hline 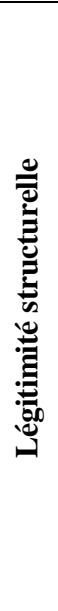 & $\begin{array}{l}\text { « Le pilotage c'est un } \\
\text { petit groupe. Il faut, } \\
\text { comme tous les } \\
\text { pilotages, que ce soit un } \\
\text { petit groupe avec } \\
\text { communication } \\
\text { permanente ». } \\
\text { «C'est la preuve qu'on } \\
\text { est arrivé à faire } \\
\text { comprendre le } \\
\text { fonctionnement. Il y a } \\
\text { une auto-organisation ». } \\
\text { « Le principal problème } \\
\text { c'est l'adhésion ». }\end{array}$ & $\begin{array}{l}\text { «Soit il faut réfléchir } \\
\text { à comment motiver } \\
\text { les enseignants- } \\
\text { chercheurs à intégrer, } \\
\text { dans leurs missions ce } \\
\text { gage de qualité. Soit } \\
\text { créer une cellule } \\
\text { qualité [...] comme il } \\
\text { y en a dans les } \\
\text { entreprises, qui } \\
\text { prenne certaines } \\
\text { tâches aux } \\
\text { enseignants- } \\
\text { chercheurs ». } \\
\text { « Il y a un manque } \\
\text { d'adhésion, quand } \\
\text { même ». }\end{array}$ & & $\begin{array}{c}\text { «Qualicert a } \\
\text { permis une } \\
\text { meilleure } \\
\text { organisation de } \\
\text { l'IAE ». } \\
\text { «C'est une } \\
\text { continuité ». }\end{array}$ & $\begin{array}{l}\text { «On a organisé } \\
\text { des groupes de } \\
\text { travail pour faire } \\
\text { ensemble les } \\
\text { fiches } \\
\text { d'évaluation. Je } \\
\text { trouve que ce } \\
\text { procédé est très } \\
\text { bien. On ne nous } \\
\text { impose rien ». }\end{array}$ \\
\hline
\end{tabular}

Le tableau montre que la mise en place de Qualicert a impliqué certains changements structurels. L'équipe de direction insiste sur un pilotage par un petit groupe de personnes soutenu par une diffusion des pratiques au sein de l'IAE aboutissant à l'auto-organisation de certains services pour atteindre les objectifs de qualité.

L'autre élément mentionné est la nécessaire et difficile adhésion de certains acteurs et notamment des enseignants-chercheurs. Cette adhésion est d'autant plus difficile à obtenir que nous sommes dans une administration publique régie par ses propres règles et contraintes telle que la difficulté de fournir des compensations financières pour motiver les parties prenantes. Les responsables de formation soulignent le fait que la structure actuelle n'est peut être pas adéquate et mentionnent la possibilité de créer une cellule dédiée à la qualité. Finalement, les responsables administratifs et les secrétaires pédagogiques pensent que Qualicert a permis d'améliorer la structure générale de l'organisation. De plus, les secrétaires pédagogiques ont également pris l'initiative de créer des équipes de travail afin d'atteindre les objectifs de qualité fixés par Qualicert.

Enfin, la légitimité morale peut également porter sur la légitimité personnelle (Tableau 8, p.12). Celle-ci se rapporte à la personne qui représente la démarche Qualicert au sein de l'IAE. 
Tableau 8 - Synthèse de la légitimité personnelle

\begin{tabular}{|c|c|c|c|c|c|}
\hline & $\begin{array}{c}\text { Groupe } 1 \\
\text { DirStr }\end{array}$ & $\begin{array}{c}\text { Groupe } 2 \\
\text { DirFor }\end{array}$ & $\begin{array}{c}\text { Groupe } 3 \\
\text { RI }\end{array}$ & $\begin{array}{c}\text { Groupe } 4 \\
\text { SerGen }\end{array}$ & $\begin{array}{c}\text { Groupe } 5 \\
\text { SP }\end{array}$ \\
\hline 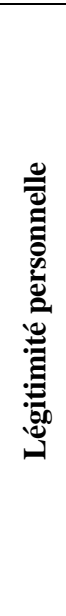 & $\begin{array}{l}\text { « Le pilote en chef } \\
\text { c'est le directeur ». } \\
\text { « Pour moi le pilote, } \\
\text { c'est S. et on est les } \\
\text { copilotes derrière ». } \\
\text { « Il y a un petit } \\
\text { groupe qui est } \\
\text { toujours très attentif } \\
\text { à ce qui se met en } \\
\text { place [...]. Et puis il } \\
\text { y a le rôle essentiel } \\
\text { de celui qui est en } \\
\text { charge. Au départ il } \\
\text { y a eu D. puis S. ». }\end{array}$ & $\begin{array}{c}\text { «On a quand } \\
\text { même la chance } \\
\text { extraordinaire } \\
\text { d'avoir S. qui } \\
\text { fait tout le } \\
\text { boulot ». } \\
\text { «De toute } \\
\text { façon, il faut } \\
\text { quelqu'un qui } \\
\text { ne fasse que ça. } \\
\text { Ce n'est pas } \\
\text { possible } \\
\text { sinon ». }\end{array}$ & $\begin{array}{l}\text { «En termes d'image } \\
\text { et d'identité, c'est très } \\
\text { clair, c'est S. et } \\
\text { d'ailleurs c'est une } \\
\text { des rares choses qui } \\
\text { soit claire à l'IAE ». } \\
\text { « Le responsable } \\
\text { Qualité à une } \\
\text { véritable visibilité, il } \\
\text { intervient à chaque } \\
\text { réunion et à tous les } \\
\text { niveaux. Il est très } \\
\text { facile de travailler } \\
\text { avec S. Il prend le } \\
\text { temps d'expliquer. Il } \\
\text { va droit au but. Il est } \\
\text { disponible ». }\end{array}$ & $\begin{array}{c}\text { «C'est S., c'est Mr } \\
\text { Qualicert ! ». } \\
\text { « Du moment que j'ai } \\
\text { un référent pour moi, } \\
\text { peu importe. Ça veut } \\
\text { dire que la direction a } \\
\text { jugé qu'il était } \\
\text { compétent sur tel } \\
\text { point ». }\end{array}$ & $\begin{array}{c}\text { «C'est S.». } \\
\text { « Je trouve que S. } \\
\text { travaille bien avec } \\
\text { nous parce qu'il } \\
\text { fait toujours des } \\
\text { propositions ». }\end{array}$ \\
\hline
\end{tabular}

L'ensemble des groupes a clairement identifié le représentant de la démarche qualité au sein de l'institut. Les verbatims recueillis attestent de la reconnaissance de sa légitimité. Notons toutefois que pour la direction, qui a chronologiquement désigné plusieurs responsables, la légitimité repose davantage sur la fonction que sur la personne elle-même.

\subsection{La légitimité cognitive}

La légitimité cognitive repose sur la légitimité de compréhensibilité et la légitimité explicite. La question posée par la légitimité de compréhensibilité est de savoir si l'organisation est capable d'offrir à ses parties prenantes, internes dans notre étude, des explications et des modèles qui la rendent déchiffrable. Le tableau ci-dessous présente une synthèse des verbatims associés à la légitimité de compréhensibilité (Tableau 9, p.12).

Tableau 9 - Synthèse de la légitimité de compréhensibilité

\begin{tabular}{|c|c|c|c|c|c|}
\hline & $\begin{array}{c}\text { Groupe } 1 \\
\text { DirStr }\end{array}$ & $\begin{array}{c}\text { Groupe } 2 \\
\text { DirFor }\end{array}$ & $\begin{array}{c}\text { Groupe } 3 \\
\text { RI }\end{array}$ & $\begin{array}{c}\text { Groupe } 4 \\
\text { SerGen }\end{array}$ & $\begin{array}{c}\text { Groupe } 5 \\
\text { SP }\end{array}$ \\
\hline 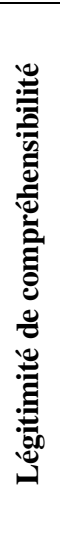 & $\begin{array}{c}\text { «C'est une } \\
\text { certification imposée } \\
\text { par le réseau ». } \\
\text { «Le monde } \\
\text { universitaire vit en } \\
\text { vase clôt ». } \\
\text { «C'est la seule } \\
\text { évaluation dans le } \\
\text { monde universitaire } \\
\text { qui est faite par des } \\
\text { gens qui ne sont pas } \\
\text { nos pairs...». }\end{array}$ & $\begin{array}{l}\text { «C'est une } \\
\text { certification }(. . .) \text {, } \\
\text { qui est censée, } \\
\text { théoriquement, être } \\
\text { un gage de qualité } \\
\text { de l'enseignement } \\
\text { supérieur». }\end{array}$ & $\begin{array}{l}\text { «Cela [Qualicert] } \\
\text { doit faire partie } \\
\text { d'une stratégie } \\
\text { donnée par l'IAE } \\
\text { qui en fasse un } \\
\text { point fort } » .\end{array}$ & $\begin{array}{c}\text { «[Qualicert] } \\
\text { impose les règles, il } \\
\text { faut suivre, mais ça } \\
\text { améliore quand } \\
\text { même, c'est un bien, } \\
\text { ça améliore tout ». } \\
\text { «C'est vrai qu'on } \\
\text { est toujours en train } \\
\text { de dire "qu'est-ce } \\
\text { qu'on a oublié ?" } \\
\text { mais après c'est } \\
\text { aussi [Qualicert] } \\
\text { une manière de } \\
\text { rappeler les } \\
\text { choses ». }\end{array}$ & $\begin{array}{c}\text { «C'est la } \\
\text { qualité. C'est la } \\
\text { norme française } \\
\text { des études ». } \\
\text { «Pour moi, } \\
\text { Qualicert c'est } \\
\text { un gage de } \\
\text { qualité, c'est la } \\
\text { preuve que tout } \\
\text { est géré selon } \\
\text { des } \\
\text { procédures ». }\end{array}$ \\
\hline
\end{tabular}

Pour la direction de la structure, la légitimité de compréhensibilité de Qualicert repose essentiellement sur son aspect d'évaluation extérieure à l'institution et à l'Université. D'un côté, l'obligation récemment instaurée par le réseau des IAE joue un rôle prépondérant pour cette forme de légitimité renvoyant à un modèle d'acceptation par la communauté. D'un autre côté, Qualicert est d'autant plus légitime qu'il est lié à un organisme extérieur. 
Les directeurs de formation ont une vision tout autre. Ces derniers ont insisté sur la distinction entre l'enseignement et la recherche. Sa légitimité de compréhensibilité provient de l'outil luimême bien qu'ils notent que celui-ci manque de notoriété.

Les relations internationales insistent sur la nécessité de lier la certification Qualicert à la stratégie de l'institut pour augmenter la cohérence globale de l'ensemble. Ils ont besoin que l'outil soit reconnu à un niveau international pour pouvoir valoriser leur formation. La légitimité de compréhensibilité n'existe pas encore réellement mais peut être envisagée et construite en suivant des axes déjà explicitement exprimés et facilitant son appropriation.

La légitimité de compréhensibilité pour les services généraux est fondée sur une double perspective fonctionnelle : cette certification explique l'amélioration de l'organisation des services mais représente aussi un outil de remémoration important.

Quant aux secrétaires pédagogiques, elles voient en Qualicert «la norme française des études ». L'explication plausible de l'utilisation de cet outil ne peut pas trouver de meilleure légitimité puisqu'il semble être accepté comme la référence nationale certifiant la qualité des études supérieures française.

Enfin, la légitimité cognitive repose également sur la légitimité explicite (Tableau 10, p.13). Les verbatims associés à celle-ci sont présentés dans le tableau ci-après.

Tableau 10 - Synthèse de la légitimité explicite

\begin{tabular}{|c|c|c|c|c|c|}
\hline & $\begin{array}{c}\text { Groupe } 1 \\
\text { DirStr }\end{array}$ & $\begin{array}{c}\text { Groupe } 2 \\
\text { DirFor }\end{array}$ & $\begin{array}{c}\text { Groupe } 3 \\
\text { RI }\end{array}$ & $\begin{array}{c}\text { Groupe } 4 \\
\text { SerGen }\end{array}$ & $\begin{array}{c}\text { Groupe } 5 \\
\text { SP }\end{array}$ \\
\hline 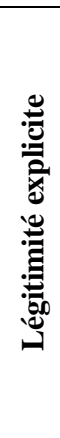 & $\begin{array}{l}\text { «Ce que l'on vend c'est le label } \\
\text { IAE pas le label IAE de Nice. } \\
\text { D'où l'intérêt du réseau, c'est } \\
\text { que tous les IAE forment les } \\
\text { étudiants avec la même } \\
\text { qualité ». } \\
\text { «C'est quelque chose qu'on a } \\
\text { tous en commun que ça soit } \\
\text { Nice, Lille ou autre. C'est } \\
\text { surtout que les étudiants soient } \\
\text { formés de la même façon et } \\
\text { pour le même objectif ». }\end{array}$ & & $\begin{array}{l}\text { « La quête de } \\
\text { qualité est une } \\
\text { stratégie à } \\
\text { long terme ». }\end{array}$ & $\begin{array}{l}\text { «Qualicert a permis } \\
\text { une meilleure } \\
\text { organisation parce que } \\
\text { l'objectif c'était que } \\
\text { l'IAE grandisse, et sans } \\
\text { Qualicert ça aurait été } \\
\text { beaucoup plus } \\
\text { compliqué ». } \\
\text { «Qualicert a donné la } \\
\text { capacité à l'IAE de } \\
\text { mieux s'organiser pour } \\
\text { grandir ». }\end{array}$ & $\begin{array}{l}\text { « Un acquis et } \\
\text { un atout. Ça } \\
\text { donne un } \\
\text { canevas. } \\
\text { Qualicert est } \\
\text { bien utile } \\
\text { quand les } \\
\text { collègues ne } \\
\text { sont pas } \\
\text { disponibles ». }\end{array}$ \\
\hline
\end{tabular}

Pour la direction de la structure, on peut considérer comme acquis que les IAE «forment les étudiants avec la même qualité ». À ce titre, le référentiel de Qualicert est un support permettant d'atteindre cet objectif de formation et qui représente «le minimum vital pour qu'un IAE tourne correctement ». Les directeurs de formation, a contrario, ne considèrent pas que Qualicert soit explicite. Pour les relations internationales, Qualicert est considéré comme explicitement légitime car ils estiment que c'est une stratégie à long terme. Les services généraux partagent ce point de vue. Pour ces derniers, la légitimité explicite est fondée sur l'amélioration de l'organisation dans une optique d'agrandissement de la structure. Pour les secrétaires pédagogiques la légitimité explicite est construite sur l'utilité de Qualicert, à long terme, dans leur travail au quotidien.

\section{Discussion des résultats et conclusion}

L'objectif principal de notre recherche, en tant que travail préalable à la construction sociale d'un outil de gestion, est de comprendre la légitimité de celui-ci par les différentes parties prenantes internes concernées et de proposer un support managérial qui permet de faciliter l'appropriation d'un outil de gestion spécifique : la démarche Qualicert au sein d'un Institut d'Administration des Entreprises. Grâce aux éléments issus de notre revue de la littérature, nous considérons que l'appropriation de la démarche qualité est liée à la légitimation de cet 
outil de gestion en interne (Buisson 2006). À travers le cadre théorique proposé par Suchman (1995), nous avons analysé la construction de la légitimité de l'outil Qualicert pour les différentes parties prenantes d'un IAE. De ce point de vue, nous avons pu remarquer que la légitimité de l'outil Qualicert est envisagée différemment selon les parties prenantes.

Cela nous permet de proposer une série d'applications pratiques pouvant favoriser l'appropriation d'un outil de gestion. Avant de présenter ces intérêts managériaux, il faut toutefois distinguer les différents légitimateurs auxquels ils s'adressent. Si les applications relatives aux parties prenantes constituées par les directeurs de formation, les relations internationales, les services généraux et les secrétaires pédagogiques s'adressent à la direction de la structure en tant que légitimateur de l'outil en interne ; les applications proposées pour la direction de la structure s'adressent à des parties prenantes externes jouant le rôle de légitimateur auprès de ce groupe, à savoir : le réseau des IAE voire la société en charge de la certification Qualicert. L'ensemble de ces applications est présenté dans le tableau ci-après (Erreur ! Source du renvoi introuvable., p.15).

Concernant la légitimité pragmatique, nous pouvons voir que, dans l'ensemble, l'outil Qualicert apporte, d'une façon ou d'une autre, des bénéfices personnels aux parties prenantes. Plus précisément, nous avons pu voir que l'outil Qualicert a permis de faciliter l'ensemble des échanges pour toutes les parties prenantes internes, excepté le groupe des relations internationales. Ainsi, la direction peut s'appuyer sur le référentiel dans ses échanges avec les différents acteurs. En fonction des parties prenantes, elle pourra insister sur le côté managérial de l'outil (pour les directeurs de formation) ou bien sur la façon dont il pourra faciliter les échanges (pour les secrétaires pédagogiques et les services généraux). L'outil a également été mis en place par la direction avec le souci de tenir compte de l'influence de toutes les parties prenantes dans ses phases de mise en place et d'amélioration. Les directeurs de formation ont ressenti un certain manque de concertation préalable au choix et à la mise en place de l'outil. Il convient donc de les intégrer dès la phase préparatoire. Alors que les directeurs de formation souhaitent avoir une influence sur l'ensemble du processus, les services généraux et les secrétaires pédagogiques devraient davantage être intégrés dans les prises de décision en fonction de leur domaine de compétence. La légitimité d'influence pour les relations internationales pourrait être favorisée si l'outil avait une visée internationale. Il convient néanmoins de les consulter en amont sur leurs attentes. Enfin, la légitimité dispositionnelle n'a été mentionnée que par le groupe des relations internationales. Cela s'explique par la nature de ce type de légitimité qui, selon nous, s'applique plus facilement à une organisation qu'à un outil. Pour le groupe qui la mentionne, l'outil Qualicert est un substitut de l'équipe de direction.

Concernant la légitimité morale, l'on se rend compte que toutes les parties prenantes, excepté le groupe des relations internationales, considèrent que l'outil contribue au bien-être de l'organisation dans son ensemble. Si l'on s'intéresse à la légitimité des résultats de Qualicert, une stratégie à long terme et une harmonisation entre les différents IAE de France sont les objectifs prioritaires valorisés par la direction de la structure. Pour les directeurs de formation, il convient d'insister sur les conséquences positives de Qualicert dans le cadre des missions d'enseignements. Pour les services généraux et les secrétaires pédagogiques, les effets concrets de Qualicert sont de deux ordres : interne et externe. D'une part, Qualicert permet d'apporter une valeur ajoutée vis-à-vis des étudiants. D'autre part, il facilite leur travail quotidien. L'adaptation sociale des procédures liées à l'outil est reconnue par la quasi-totalité des parties prenantes. Ainsi, pour la direction de la structure, Qualicert est moins perçue comme une fin que comme un moyen de diffuser une démarche qualité totale dans l'ensemble de la structure. Pour les directeurs de formation, l'avantage de l'outil Qualicert, en tant que démarche qualité, est qu'il fait lui aussi l'objet d'un processus d'amélioration continue de ces 
procédures. Quant aux services généraux et aux secrétaires pédagogiques, l'outil permet d'harmoniser les pratiques de travail dans une optique de « bonne chose à faire ».

Tableau 11 - Applications managériales

\begin{tabular}{|c|c|c|c|c|c|}
\hline & $\begin{array}{l}\text { Groupe } 1 \\
\text { DirStr }\end{array}$ & $\begin{array}{l}\text { Groupe } 2 \\
\text { DirFor }\end{array}$ & $\begin{array}{l}\text { Groupe } 3 \\
\text { RI }\end{array}$ & $\begin{array}{l}\text { Groupe } 4 \\
\text { SerGen }\end{array}$ & $\begin{array}{l}\text { Groupe } 5 \\
\text { SP }\end{array}$ \\
\hline \multicolumn{6}{|l|}{$\begin{array}{l}\text { Légitimité } \\
\text { pragmatique }\end{array}$} \\
\hline $\begin{array}{l}\text { Légitimité } \\
\text { d'échange }\end{array}$ & $\begin{array}{l}\text { S'appuyer sur le } \\
\text { référentiel pour } \\
\text { justifier les } \\
\text { décisions. }\end{array}$ & $\begin{array}{l}\text { Présenter l'aspect } \\
\text { managérial de } \\
\text { l'outil dans le cadre } \\
\text { des échanges avec } \\
\text { les étudiants et les } \\
\text { enseignants. }\end{array}$ & & \multicolumn{2}{|c|}{$\begin{array}{l}\text { Présenter Qualicert comme un outil } \\
\text { d'aide aux échanges quotidiens et de } \\
\text { facilitation des relations } \\
\text { hiérarchiques. }\end{array}$} \\
\hline $\begin{array}{l}\text { Légitimité } \\
\text { d'influence }\end{array}$ & $\begin{array}{l}\text { Impliquer les } \\
\text { services dans la } \\
\text { mise en place de la } \\
\text { démarche. }\end{array}$ & $\begin{array}{l}\text { Intégrer en amont } \\
\text { les directeurs de } \\
\text { formation. }\end{array}$ & & \multicolumn{2}{|c|}{$\begin{array}{l}\text { Implication en amont dans les } \\
\text { décisions liées à leurs domaines de } \\
\text { compétence. }\end{array}$} \\
\hline $\begin{array}{c}\text { Légitimité } \\
\text { dispositonnelle }\end{array}$ & & & $\begin{array}{l}\text { elais } \\
\text { ction } \\
\text { ies } \\
\text { s. }\end{array}$ & & \\
\hline \multicolumn{6}{|l|}{$\begin{array}{l}\text { Légitimité } \\
\text { morale }\end{array}$} \\
\hline $\begin{array}{c}\text { Légitimité } \\
\text { conséquentielle }\end{array}$ & $\begin{array}{c}\text { Stratégie de long } \\
\text { terme, } \\
\text { harmonisation entre } \\
\text { les IAE de France. }\end{array}$ & $\begin{array}{l}\text { Présenter l'outil } \\
\text { comme un atout } \\
\text { essentiel pour les } \\
\text { missions } \\
\text { d'enseignement. }\end{array}$ & & \multicolumn{2}{|c|}{$\begin{array}{c}\text { Insister sur la valeur ajoutée de } \\
\text { Qualicert vis-à-vis du public externe } \\
\text { (étudiants). } \\
\text { Présenter Qualicert comme un outil } \\
\text { d'aide au travail quotidien. }\end{array}$} \\
\hline $\begin{array}{l}\text { Légitimité } \\
\text { procédurale }\end{array}$ & $\begin{array}{l}\text { Dépasser l'outil } \\
\text { pour insister sur une } \\
\text { démarche qualité } \\
\text { globale. }\end{array}$ & $\begin{array}{l}\text { Insister sur le } \\
\text { processus } \\
\text { d'amélioration } \\
\text { continue. }\end{array}$ & & \multicolumn{2}{|c|}{$\begin{array}{l}\text { Présenter Qualicert comme un guide } \\
\text { des «bonnes pratiques » de travail. }\end{array}$} \\
\hline $\begin{array}{l}\text { Légitimité } \\
\text { structurelle }\end{array}$ & $\begin{array}{l}\text { Favoriser une auto- } \\
\text { organisation. } \\
\text { Susciter l'adhésion. } \\
\text { Désigner des } \\
\text { interlocuteurs } \\
\text { permanents. }\end{array}$ & $\begin{array}{l}\text { Créer une cellule } \\
\text { qualité. } \\
\text { Susciter l'adhésion } \\
\text { et la motivation. }\end{array}$ & & $\begin{array}{l}\text { Insister sur les } \\
\quad \text { qualités } \\
\text { organisationnel- } \\
\text { les de Qualicert. }\end{array}$ & $\begin{array}{l}\text { Favoriser le } \\
\text { travail en équipe } \\
\text { et l'auto- } \\
\text { organisation. }\end{array}$ \\
\hline $\begin{array}{l}\text { Légitimité } \\
\text { personnelle }\end{array}$ & \multicolumn{5}{|c|}{$\begin{array}{l}\text { Déléguer la démarche qualité à une unique personne, en lien direct avec la direction, qui sera le } \\
\text { représentant Qualité de l'institut ou de la cellule dédiée à cette démarche. }\end{array}$} \\
\hline \multicolumn{6}{|l|}{$\begin{array}{l}\text { Légitimité } \\
\text { cognitive }\end{array}$} \\
\hline $\begin{array}{l}\text { Légitimité de } \\
\text { compréhensibilité }\end{array}$ & $\begin{array}{l}\text { Reconnaissance par } \\
\text { le réseau des IAE. } \\
\text { Aspect positif d'une } \\
\text { évaluation } \\
\text { extérieure. }\end{array}$ & $\begin{array}{l}\text { Insister sur la } \\
\text { valorisation } \\
\text { apportée par } \\
\text { Qualicert pour } \\
\text { l'enseignement } \\
\text { supérieur. }\end{array}$ & $\begin{array}{l}\text { Focaliser sur } \\
\text { l'aspect } \\
\text { stratégique de } \\
\text { l'outil. }\end{array}$ & $\begin{array}{l}\text { Insister sur les } \\
\text { fonctions } \\
\text { «amélioration » } \\
\text { et } \\
\text { «remémoration » } \\
\text { de l'outil. }\end{array}$ & $\begin{array}{c}\text { Insister la } \\
\text { valorisation } \\
\text { apportée par } \\
\text { Qualicert pour } \\
\text { l'enseignement } \\
\text { supérieur. }\end{array}$ \\
\hline $\begin{array}{l}\text { Légitimité } \\
\text { explicite }\end{array}$ & $\begin{array}{l}\text { Renforcer l'image } \\
\text { de Qualicert à } \\
\text { travers le } \\
\text { « réseau des IAE ». }\end{array}$ & & $\begin{array}{l}\text { Véhiculer une } \\
\text { image de } \\
\text { Qualicert qui dure } \\
\text { dans le temps. }\end{array}$ & $\begin{array}{l}\text { Renforcer le } \\
\text { caractère } \\
\text { organisationnel de } \\
\text { Qualicert dans } \\
\text { une optique de } \\
\text { développement de } \\
\text { l'institut. }\end{array}$ & $\begin{array}{l}\text { Caractère utile } \\
\text { de Qualicert } \\
\text { pour le travail } \\
\text { au quotidien. }\end{array}$ \\
\hline
\end{tabular}

Si l'on s'intéresse aux applications relatives à la légitimité structurelle, l'on s'aperçoit que la quasi-totalité des parties prenantes considère que le pilotage de Qualicert permet d'atteindre, de manière collective, la certification qualité de façon appropriée et adéquate. Pour la 
direction de la structure, il importe que la structure de pilotage soit durable et constituée d'un groupe de personnes suscitant l'adhésion générale et favorisant l'auto-organisation de l'ensemble des parties prenantes internes. Les directeurs de formations rejoignent la perspective de la direction de la structure en insistant sur la nécessaire création d'une cellule qualité. Toutefois l'adhésion et la motivation de ce groupe peuvent s'avérer difficiles à obtenir en raison du caractère «philanthropique » de leur participation à la démarche qualité. Les services généraux et les secrétaires pédagogiques sont sensibles aux effets positifs de Qualicert sur l'organisation générale de l'institut. Plus spécifiquement, les secrétaires pédagogiques manifestent un intérêt particulier pour la réorganisation de leur travail en équipe dans l'optique d'atteindre les objectifs fixés par Qualicert. Enfin, la légitimité des individus chargés du bon fonctionnement de l'outil Qualicert en interne est reconnue par toutes les parties prenantes. Nous constatons qu'il est essentiel qu'une personne charismatique soit responsable de la cellule qualité au sein de la structure. Celui-ci assume le rôle de référent pour toutes les parties prenantes internes.

Enfin, la légitimité cognitive de Qualicert, qui repose sur son acceptation comme allant de soi, est reconnue par la majorité des parties prenantes. La compréhensibilité de l'outil se fonde sur la production d'explication et de modèles qui le rende déchiffrable pour toutes les parties prenantes. Ainsi, la légitimité de compréhensibilité repose, pour la direction de la structure, sur la reconnaissance de l'outil par le réseau des IAE et sur le côté « externe » de l'évaluation. Pour les directeurs de formation et les secrétaires pédagogiques, la compréhensibilité de Qualicert est uniquement fondée sur la valorisation de l'enseignement supérieur. Les relations internationales acceptent Qualicert pour le rapport qu'il entretient avec la stratégie de l'institut. Quant aux services généraux, la justification de l'existence de Qualicert repose sur sa double fonction d'amélioration et de remémoration du travail à effectuer.

Enfin, la légitimité explicite, qui repose sur l'institutionnalisation de l'outil et sa pérennité, est reconnue par toutes les parties prenantes, excepté par les directeurs de formation.

Pour la direction de la structure, la pérennité de Qualicert repose sur la constance et la cohésion du réseau des IAE. Les relations internationales jugent que la légitimité explicite s'appuie sur l'existence d'une stratégie. Pour les services généraux, la pérennité de Qualicert ne se justifie qu'à travers la recherche constante du développement de l'institut. Enfin, les secrétaires pédagogiques estiment que Qualicert perdurera car il représente tout simplement les bonnes pratiques de travail.

Pour autant, les résultats sont à nuancer. Si ces derniers montrent que l'outil Qualicert est perçu comme légitime par l'ensemble des groupes, il convient d'exposer les réserves mentionnées par deux groupes. Le groupe des directeurs de formation note que Qualicert «ne s'intègre pas très bien avec nos objectifs individuels, en termes de carrière ». En effet, pour les directeurs de formation interrogés, Qualicert concerne une partie de leurs tâches administratives, or celles-ci sont les moins valorisées dans leur évaluation professionnelle. Pour le groupe des relations internationales, la construction de la légitimité de l'outil paraît plus difficile. Pour ce groupe, Qualicert représente «une série de contraintes, un décret d'application avec des règles à suivre pour mettre en place une loi ». Cela s'explique en partie par le fait que ce groupe ne s'est pas sentit impliqué : «je n'ai pas été consulté dans la mise en place de Qualicert». Des éléments de contexte particuliers peuvent expliquer la difficulté de ce groupe à construire cette légitimité. De manière générale, les verbatims font ressortir un sentiment d'exclusion : "je souffre pas mal de l'exil au troisième étage. Ça éclate la structure et l'établissement ». De plus, les missions propres au pôle international ne leur permettent pas de valoriser Qualicert dont la couverture pour l'enseignement supérieur est uniquement nationale : "au niveau international, cela n'a pas de visibilité et les partenaires me demandent de quoi il s'agit». 
Le cas du groupe des relations internationales nous montre que la mise en place d'un outil de gestion n'est réalisable "que dans une relation de négociation et d'échange, pas par imposition. La réussite n'a lieu que dans la mesure où tous ceux qui ont à faire avec ces changements sont considérés comme de véritables acteurs dans leur réseau de relations» (Bernoux, 2010, p.240-241). Cela nous conduit à envisager un cadre théorique alternatif pour étudier l'appropriation de Qualicert au sein d'un IAE. En effet, la sociologie de la traduction et la théorie de l'acteur-réseau (Callon, 1986 ; Akrich, Callon, et Latour, 2006) peuvent constituer un support pertinent pour analyser l'appropriation d'un outil de gestion, par différentes parties prenantes, au sein d'un réseau. Ce cadre théorique permettrait ainsi de mieux comprendre le comportement des acteurs concernés, les phénomènes de négociation et de construction de compromis, et finalement d'identifier les facteurs qui vont favoriser ou freiner l'appropriation de l'outil.

Pour conclure, nous rappelons que l'objet de cet article était d'analyser l'appropriation d'un outil de gestion à travers le prisme de la légitimité. Nous avons pu voir, à travers l'étude d'un cas particulier : celui d'un outil de gestion de la qualité au sein d'un IAE que l'appropriation d'un outil peut s'analyser à travers la construction de sa légitimité par les différentes parties prenantes concernées. L'article permet de montrer que le cadre théorique de la légitimité de Suchman (1995), initialement développé dans l'objectif d'analyser la légitimité au sein des organisations, peut être mobilisé de manière pertinente pour l'analyse d'un outil de gestion de la qualité au sein d'un institut d'enseignement supérieur public. Toutefois, nous notons que les sous-catégories de la légitimité développées par Suchman ne sont pas aussi hermétiques que ce que propose l'auteur. En effet, les trois grands types de légitimité sont facilement identifiables dans l'analyse de nos données tandis que les sous-catégories se confondent parfois. Cette recherche, dont les limites sont inhérentes à sa nature exploratoire et qualitative, mérite d'être prolongée par des études au sein d'autres IAE pour confirmer les éléments de la construction de la légitimité selon Suchman (1995) auprès des parties prenantes internes. Dans cette optique, il conviendrait d'y intégrer des éléments relatifs à la culture des organisations. Enfin, il serait envisageable de prolonger cette étude relative aux parties prenantes internes à l'organisation par une étude de la construction de la légitimé de l'outil de gestion par les prenantes externes incluant les relations entre le cas de l'IAE étudié, le réseau des IAE et l'organisme de certification qualité.

\section{Bibliographie}

Akrich M., Callon M. et Latour, B. (2006), Sociologie de la traduction. Textes fondateurs, Presses de l'Ecole des Mines de Paris, Paris.

Bernoux P. (2010), «L'anthropologie dans l'enseignement en management: la reconnaissance sociale », Management \& Avenir, Vol. 6, `36, p. 231-244.

Buisson M.-L. (2006), «Diffusion et appropriation des outils de gestion : l'apport de la théorie néo-institutionnelle » in A. Grimand (Ed.), L'appropriation des outils de gestion : vers de nouvelles perspectives théoriques? Publications de l'Université de Saint-Etienne, SaintEtienne, p. 157-173.

Callon M. (1986), «Éléments pour une sociologie de la traduction, la domestication des coquilles saint-jacques et des marins-pêcheurs dans la baie de Saint-Brieuc », L'Année Sociologique, Vol. 36, p. 169-208.

Chevalier G. (2009), Eléments de management public : Le management public par la qualité, Editions Afnor, Paris.

David A. (1998), « Outils de gestion et dynamique du changement », Revue Française de Gestion, septembre-octobre, $\mathrm{N}^{\circ} 120$, p. 44-59. 
David, A. (2003), «Etude de cas et généralisation scientifique en sciences de gestion », Revue Sciences de Gestion, N³9, p. 139-166.

De Benedetti A. et Philippe D. (2011), «Stratégie de communication environnementale et construction de légitimité : Le cas de PSA Peugeot Citroën », Décisions Marketing, JanvierMars, $\mathrm{N}^{\circ} 61$, p. 67-71.

De Vaujany F.X. (2006), «Pour une théorie de l'appropriation des outils de gestion : vers un dépassement de l'opposition conception-usage », Management \& Avenir, Vol. 3, N 9, p. 109126.

Demers C. (2003), «L'entretien » in Y. Giordano (Ed.), Conduire un projet de recherche, Editions EMS, Paris.

Deschenaux F. (2007), « Guide d'introduction au logiciel QSR Nvivo 7. ARQ », Les cahiers pédagogiques de l'Association pour la recherche qualitative.

Duchesne S. et Haegel F. (2008), L'entretien collectif: l'enquête et ses méthodes, Editions A. Colin, Paris.

DiMaggio P. J. et Powell W. W. (1983), « The iron cage revisited: Institutional isomorphism and collective rationality in organizational fields », American Sociological Review, Vol.48, $\mathrm{N}^{\circ}$ 2, p. 147-160.

DiMaggio P. J. et Powell W. W. (1991), The new institutionalism in organizational analysis, The University of Chicago press, Chicago.

Fontana A. et Frey J. H. (1994), Interviewing: The art of science in Handbook of qualitative research, Sage Publications, Thousand Oaks.

Grenard A. (1996), «Normalisation, certification : quelques éléments de définition », Revue d'économie industrielle, Vol. 75, $1^{\mathrm{er}}$ trimestre, p. 45-60.

Grimand A. (2006), «Introduction : l'appropriation des outils de gestion, entre rationnalité instrumentale et construction du sens » in A. Grimand (Ed.), L'appropriation des outils de gestion: vers de nouvelles perspectives théoriques? Publications de l'Université de SaintEtienne, Saint-Etienne, p.13-27.

Hatchuel A. et Weil B. (1992), L'expert et le système, Economica, Paris.

Johnson A. (1996), «It's good to talk: the focus group and the sociological imagination ». The Sociological Review, Vol. 44, N³, p. 517-538.

Laufer R. (2008), «Les institutions du management : légitimité, organisation et nouvelle rhétorique » in A. David, A. Hatchuel et R. Laufer (Eds.), Les nouvelles fondations des

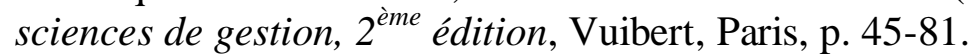

Merton R. K., Lowenthal M. F. et Kendall P. L. (1990), The focused interview: A manual of problems and procedures, Free Press.

Morgan D. L. (1997), Focus groups as qualitative research, Sage Publications, Thousand Oaks.

Oliver C. (1991), «Strategic responses to institutional processes », Academy of Management Review, Vol.16, p.145-179.

Parsons T. (1960), Structure and process in modem societies, Free Press, Glencoe, IL.

Pettigrew A. (1979), " On studying organizational cultures », Administrative Science Quarterly, Vol. 24, p. 570-581.

Phillips N., Lawrence T.B. et Hardy C. (2004), « Discourses and institutions », Academy of Management Review, Vol. 29, N4, p.635-652.

Rolland S. (2009), «Un bilan de 20 ans de certification des systèmes de management de la qualité : les apports perçus de la certification ISO 9000 par les managers », Management \& Avenir, Vol. 9, №29, p. 31-51.

Scott W. R. (1992), Organizations: Rational, natural and open systems, $3^{\text {ème }}$ édition, Prentice Hall, Englewood Cliffs, NJ. 
Suchman M.C. (1995), " Managing legitimacy: strategic and institutional approaches », Academy of Management Review, Vol. 20, N³, p. 571-610.

Thiétart R. (2007), Méthodes de recherche en management, Dunod, Paris. 